\title{
On a product-type operator between Hardy and $\alpha$-Bloch spaces of the upper half-plane
}

\author{
Stevo Stevici ${ }^{1,2^{*}}$ and Ajay K. Sharma ${ }^{3}$
}

"Correspondence: sstevic@ptt.rs 'Mathematical Institute of the Serbian Academy of Sciences, Beograd, Serbia

${ }^{2}$ Department of Medical Research, China Medical University Hospital, China Medical University, Taichung, Taiwan, Republic of China

Full list of author information is available at the end of the article

\begin{abstract}
Recently we have introduced a product-type operator and studied it on some spaces of analytic functions on the unit disc. Here we start investigating the operator on the space of analytic functions on the upper half-plane. We characterize the boundedness and compactness of the operator between Hardy and $\alpha$-Bloch spaces on the domain.
\end{abstract}

MSC: Primary 47B38; 46E10; secondary 30D45

Keywords: Product-type operator; Hardy space; Bloch space; Boundedness; Compactness; Upper half-plane

\section{Introduction}

Let $\mathbb{D}$ be the unit disc in the complex plane $\mathbb{C}, \Pi^{+}=\{z \in \mathbb{C}: \Im z>0\}$ the upper half-plane in $\mathbb{C}$, and $\widehat{\Pi^{+}}=\overline{\Pi^{+}} \cup\{\infty\}$. Let $\Omega$ be a domain in $\mathbb{C}$. We denote by $H(\Omega)$ the space of all analytic functions on $\Omega$ and by $S(\Omega)$ the class of all analytic self-maps of $\Omega$.

For $0<p<\infty$, the Hardy space of $\Pi^{+}$, denoted by $H^{p}\left(\Pi^{+}\right)$, consists of all $f \in H\left(\Pi^{+}\right)$such that

$$
\|f\|_{H^{p}\left(\Pi^{+}\right)}^{p}=\sup _{y>0} \int_{-\infty}^{\infty}|f(x+i y)|^{p} d x<\infty
$$

For $1 \leq p<\infty, H^{p}\left(\Pi^{+}\right)$is a Banach space.

Let $\alpha>0$. The $\alpha$-Bloch or Bloch-type space on $\Pi^{+}$, denoted by $\mathcal{B}^{\alpha}\left(\Pi^{+}\right)$, consists of all $f \in H\left(\Pi^{+}\right)$such that

$$
\|f\|_{\mathcal{B}^{\alpha}\left(\Pi^{+}\right)}:=|f(i)|+\sup _{z \in \Pi^{+}}(\Im z)^{\alpha}\left|f^{\prime}(z)\right|<\infty
$$

With the norm (1), the $\alpha$-Bloch space is a Banach space. For Bloch-type spaces on various domains and operators on them, see, for example, [1-13] and the references therein. For a natural extension of Bloch-type spaces and for Zygmud-type spaces, see [14-16].

For $\varphi \in S(\Omega)$, the composition operator $C_{\varphi}$ is the linear operator defined by

$$
C_{\varphi}(f)(z)=(f \circ \varphi)(z) \quad \text { for } f \in H(\Omega) .
$$

(c) The Author(s) 2018. This article is distributed under the terms of the Creative Commons Attribution 4.0 International License (http://creativecommons.org/licenses/by/4.0/), which permits unrestricted use, distribution, and reproduction in any medium, provided you give appropriate credit to the original author(s) and the source, provide a link to the Creative Commons license, and indicate if changes were made. 
For $\psi \in H(\Omega)$, the multiplication operator $M_{\psi}$ is defined on $H(\Omega)$ by

$$
M_{\psi} f(z)=\psi(z) f(z) \quad \text { for } f \in H(\Omega) .
$$

The product of these two operators

$$
W_{\varphi, \psi}=M_{\psi} \circ C_{\varphi}
$$

is the so-called weighted composition operator.

By $D$ we denote the differentiation operator, that is,

$$
D f=f^{\prime}, \quad f \in H(\Omega) .
$$

These concrete operators, along with some integral-type ones, are among those considerably studied, during the last five decades, on spaces of analytic functions on various domains in $\mathbb{C}$ or domains in the complex-vector space $\mathbb{C}^{n}$. Majority of the papers on the operators are devoted to investigating them on spaces of analytic functions on $\mathbb{D}$. Much less papers consider the operators on spaces of analytic functions on other domains, including the upper half-plane. Even for some popular operators, such as the composition ones, up to the end of the previous century, there are no many papers on popular spaces such as $H^{p}\left(\Pi^{+}\right)$(see, e.g., [17-20] and the references therein). Hence, any new result on the spaces and operators on $\Pi^{+}$is of some interest. Regarding some operators on the mentioned spaces, let us mention that some basic results on the boundedness of composition operators from $H^{p}\left(\Pi^{+}\right)$to the classical Bloch space $\mathcal{B}\left(\Pi^{+}\right)$can be found in note [21]. For related investigations of composition or weighted composition operators on some other spaces, see [14, 15, 22-25]. Let us mention that the behavior of composition operators on spaces of analytic functions in $\Pi^{+}$is considerably different from the behavior of composition operators on spaces of analytic functions in $\mathbb{D}$. For example, every analytic self-map of $\mathbb{D}$ induces a bounded composition operator on the corresponding Hardy space, which is not always the case on the space $H^{p}\left(\Pi^{+}\right)[17,18]$.

From 1968 to 2005, experts more or less studied theoretic properties of only operators (2)-(5) and integral-type ones on spaces of analytic functions in terms of their symbols. The only product-type operator among (2)-(5) is the weighted composition operator. Since 2005, some experts started studying some other product-type operators. The first product-type operators different from weighted composition ones that attracted some attention were the products of composition and differentiation operators (see, e.g., [7, 11, 13, 26-29] and the references therein). Some generalizations of the products of composition and differentiation operators, containing iterated differentiation, have appeared soon after them (see [12, 30-33]). Around 2008, Li and Stević have initiated studying products of integral and composition operators, including in some cases the differentiation operator, on spaces of analytic functions on $\mathbb{D}$ (see, e.g., [34]), which have been considerably studied recently (see, e.g., [3, 35-40]). For some other results and related product-type operators, see, for example, [4-6, 35, 41-46].

To treat product-type operators consisting of exactly one composition, multiplication and differentiation operator in a unified manner, we have recently introduced a generalized operator and studied it on the weighted Bergman spaces (see [45] and [46]). See also papers 
$[5,42]$ on the operator on some other spaces of functions defined by

$$
T_{\psi_{1}, \psi_{2}, \varphi} f(z)=\psi_{1}(z) f(\varphi(z))+\psi_{2}(z) f^{\prime}(\varphi(z)), \quad f \in H(\mathbb{D})
$$

where $\psi_{1}, \psi_{2} \in H(\mathbb{D})$ and $\varphi \in S(\mathbb{D})$.

It is clear that operator (6) includes the composition, multiplication, differentiation, weighted composition, weighted differentiation composition, and many other concrete operators, including those in $[6,41,43]$, which are obtained by some concrete choices of the symbols $\psi_{1}, \psi_{2}$, and $\varphi$. This is one of the reasons why the operator is of some importance for investigation.

So far the operator has not been considered between spaces of analytic functions on the upper half-plane. Here we start investigating the operator on such spaces by characterizing the boundedness and compactness of the operator between the Hardy and $\alpha$-Bloch spaces on the domain, that is, of $T_{\psi_{1}, \psi_{2}, \varphi}: H^{p}\left(\Pi^{+}\right) \rightarrow \mathcal{B}^{\alpha}\left(\Pi^{+}\right)$. We provide a complete characterization of the compactness. The paper can be regarded as a continuation of our investigations in [14, 15, 22-25].

Throughout this paper, constants are denoted by $C$; they are positive and not necessarily the same at each occurrence. The notation $A \asymp B$ means that $B \lesssim A \lesssim B$, where $A \lesssim B$ means that there is a positive constant $C$ such that $A \leq C B$.

\section{Auxiliary results}

First, we quote a point evaluation lemma, which is a folklore result (see, e.g., [15, Lemma 3]).

Lemma 1 Let $p \in(0, \infty)$ and $n \in \mathbb{N}_{0}$. Then

$$
\left|f^{(n)}(z)\right| \leq C \frac{\|f\|_{H^{p}\left(\Pi^{+}\right)}}{y^{n+\frac{1}{p}}}
$$

for some positive constant $C=C(p, n)$ independent off .

The following lemma can be found in [14, Lemma 2.1].

Lemma 2 Let $p \in[1, \infty), k \in \mathbb{N}_{0}, \zeta \in \Pi^{+}$, and

$$
f_{\zeta, k}(z)=\frac{(\Im \zeta)^{k-\frac{1}{p}}}{(z-\bar{\zeta})^{k}} .
$$

Then

$$
\sup _{\zeta \in \Pi^{+}}\left\|f_{\zeta, k}\right\|_{H^{p}\left(\Pi^{+}\right)} \leq \pi^{\frac{1}{p}}
$$

To deal with the compactness of the operator $T_{\psi_{1}, \psi_{2}, \varphi}: H^{p}\left(\Pi^{+}\right) \rightarrow \mathcal{B}^{\alpha}\left(\Pi^{+}\right)$, in the lemma that follows, we give its characterization, which is typical for concrete operators between spaces of analytic functions. The thesis of Schwartz [47] is one of the first sources that presents such a characterization for the case of a concrete operator, more precisely, for a 
composition operator. It is interesting that the proof of our present lemma is more complicated than those of the corresponding lemmas that we have had so for (for example, the lemma in [24]). Hence we will present a detailed proof of the lemma.

We say that a sequence $\left(f_{n}\right)_{n \in \mathbb{N}}$ in $H^{p}\left(\Pi^{+}\right)$converges weakly to zero if it is norm bounded in $H^{p}\left(\Pi^{+}\right)$and converges to zero on compacts of $\Pi^{+}$.

Lemma 3 Let $p \geq 1, \alpha \in(0,+\infty), \psi_{1}, \psi_{2} \in H\left(\Pi^{+}\right)$, and $\varphi \in S\left(\Pi^{+}\right)$. Then $T_{\psi_{1}, \psi_{2}, \varphi}$ : $H^{p}\left(\Pi^{+}\right) \rightarrow \mathcal{B}^{\alpha}\left(\Pi^{+}\right)$is compact if and only if for any sequence $\left(f_{n}\right)_{n \in \mathbb{N}}$ weakly convergent to zero, we have

$$
\lim _{n \rightarrow \infty}\left\|T_{\psi_{1}, \psi_{2}, \varphi f_{n}}\right\|_{\mathcal{B}^{\alpha}\left(\Pi^{+}\right)}=0
$$

Proof Let the operator be compact. Suppose that $\left(f_{n}\right)_{n \in \mathbb{N}}$ weakly converges to zero. Then there are a subsequence $\left(f_{n_{k}}\right)_{k \in \mathbb{N}}$ and $g \in \mathcal{B}^{\alpha}\left(\Pi^{+}\right)$such that

$$
\lim _{k \rightarrow \infty}\left\|T_{\psi_{1}, \psi_{2}, \varphi} f_{n_{k}}-g\right\|_{\mathcal{B}^{\alpha}\left(\Pi^{+}\right)}=0
$$

Let $K \subset \Pi^{+}$be compact. Then

$$
d_{K}:=d\left(K, \partial \Pi^{+}\right)=\inf _{z \in K, x \in \mathbb{R}}|z-x|>0 .
$$

From this and from (1) we have

$$
\left|T_{\psi_{1}, \psi_{2}, \varphi} f_{n_{k}}(i)-g(i)\right| \leq\left\|T_{\psi_{1}, \psi_{2}, \varphi} f_{n_{k}}-g\right\|_{\mathcal{B}^{\alpha}\left(\Pi^{+}\right)}
$$

and

$$
\sup _{z \in K}\left|\left(T_{\psi_{1}, \psi_{2}, \varphi} f_{n_{k}}(z)-g(z)\right)^{\prime}\right| \leq \frac{1}{d_{K}}\left\|T_{\psi_{1}, \psi_{2}, \varphi} f_{n_{k}}-g\right\|_{\mathcal{B}^{\alpha}\left(\Pi^{+}\right) .} .
$$

Let $A \subset \Pi^{+}$and $A_{\varepsilon}=\left\{z \in \Pi^{+}: d(z, K) \leq \varepsilon\right\}$, where $\varepsilon>0$. Note that if $A$ is compact, then for each $\varepsilon<d_{K}$, the set $A_{\varepsilon}$ is also a compact set as bounded and closed.

On the other hand, we have

$$
f(z)=f(i)+\int_{i}^{z} f^{\prime}(\zeta) d \zeta
$$

for all $f \in H\left(\Pi^{+}\right)$and $z \in \Pi^{+}$.

Let $A \subset \Pi^{+}$and

$$
A(i)=\left\{w \in \Pi^{+}: \exists z \in A, w \in[i, z]\right\} .
$$

Note that if $A$ is compact, then $A(i)$ is also compact.

Hence from (11) we easily get

$$
|f(z)| \leq|f(i)|+\operatorname{diam}(K(i)) \sup _{w \in K(i)}\left|f^{\prime}(w)\right|
$$

for each $z \in K$. 
From (9), (10), and (12) it follows that

$$
\begin{aligned}
\sup _{z \in K}\left|T_{\psi_{1}, \psi_{2}, \varphi} f_{n_{k}}(z)-g(z)\right| \leq & \mid\left(T_{\psi_{1}, \psi_{2}, \varphi} f_{n_{k}}(i)-g(i) \mid\right. \\
& +\operatorname{diam}(K(i)) \sup _{z \in K(i)}\left|\left(T_{\psi_{1}, \psi_{2}, \varphi} f_{n_{k}}(z)-h(z)\right)^{\prime}\right| \\
\leq & \left(1+\frac{\operatorname{diam}(K(i))}{d_{K(i)}}\right)\left\|T_{\psi_{1}, \psi_{2}, \varphi} f_{n_{k}}-g\right\|_{\mathcal{B}^{\alpha}\left(\Pi^{+}\right)}
\end{aligned}
$$

From (8) and (13) it follows that

$$
T_{\psi_{1}, \psi_{2}, \varphi} f_{n_{k}}(z)-g(z) \rightrightarrows 0, \quad k \rightarrow \infty
$$

on each compact $K \in \Pi^{+}$.

Further, note that

$$
\widehat{M}_{j}:=\sup _{z \in K}\left|\psi_{j}(z)\right|<\infty, \quad j=1,2,
$$

for each compact $K$. The compactness of the set $\varphi(K)$ also implies that

$$
\max \left\{f_{n_{k}}(\varphi(z)), f_{n_{k}}^{\prime}(\varphi(z))\right\} \rightrightarrows 0, \quad k \rightarrow \infty
$$

on each compact $K \in \Pi^{+}$.

From this, since

$$
\left|T_{\psi_{1}, \psi_{2}, \varphi} f_{n_{k}}(\varphi(z))\right| \leq \widehat{M}_{1} \sup _{w \in \varphi(K)}\left|f_{n_{k}}(w)\right|+\widehat{M}_{2} \sup _{w \in \varphi(K)}\left|f_{n_{k}}^{\prime}(w)\right|
$$

it follows that

$$
T_{\psi_{1}, \psi_{2}, \varphi} f_{n_{k}}(z) \rightrightarrows 0, \quad k \rightarrow \infty
$$

on each compact $K \in \Pi^{+}$.

From (14) and (15) we obtain $g(z)=0$ for every $z \in \Pi^{+}$, since each $z \in \Pi^{+}$lies in a compact subset of $\Pi^{+}$.

Using the fact in (8), it follows that

$$
\lim _{k \rightarrow \infty}\left\|T_{\psi_{1}, \psi_{2}, \varphi} f_{n_{k}}\right\|_{\mathcal{B}^{\alpha}\left(\Pi^{+}\right)}=0
$$

Such a procedure can be applied to any subsequence of $\left(f_{n}\right)_{n \in \mathbb{N}}$, from which it follows that (7) holds.

Now assume that, for any sequence $\left(f_{n}\right)_{n \in \mathbb{N}}$ weakly convergent to zero, we have $\lim _{n \rightarrow \infty}\left\|T_{\psi_{1}, \psi_{2}, \varphi} f_{n}\right\|_{\mathcal{B}^{\alpha}\left(\Pi^{+}\right)}=0$. Let $\left.\widehat{f}_{n}\right)_{n \in \mathbb{N}}$ be a sequence of functions such that $M:=$ $\sup _{n \in \mathbb{N}}\left\|\widehat{f}_{n}\right\|_{H^{p}\left(\Pi^{+}\right)}<+\infty$. By Lemma 1 the sequence is uniformly bounded on compacts of $\Pi^{+}$, and consequently normal. Hence there are a subsequence $\left(\widehat{f}_{n_{k}}\right)_{k \in \mathbb{N}}$ and $\widehat{f} \in H\left(\Pi^{+}\right)$ such that

$$
\widehat{f}_{n_{k}}(z)-\widehat{f}(z) \rightrightarrows 0, \quad k \rightarrow \infty
$$


on each compact $K \in \Pi^{+}$. The Fatou lemma along with (17) implies $\|\widehat{f}\|_{H^{p}\left(\Pi^{+}\right)} \leq M$. Hence, the sequence $\left(\widehat{f}_{n_{k}}-\widehat{f}\right)_{k \in \mathbb{N}}$ weakly converges to zero, and consequently

$$
\lim _{k \rightarrow \infty}\left\|T_{\psi_{1}, \psi_{2}, \varphi \widehat{f}_{n_{k}}}-T_{\psi_{1}, \psi_{2}, \varphi} \widehat{f}\right\|_{\mathcal{B}^{\alpha}\left(\Pi^{+}\right)}=0,
$$

from which the compactness of the operator $T_{\psi_{1}, \psi_{2}, \varphi}: H^{p}\left(\Pi^{+}\right) \rightarrow \mathcal{B}^{\alpha}\left(\Pi^{+}\right)$follows.

\section{Boundedness and compactness of $T_{\psi_{1}, \psi_{2}, \varphi}: H^{p}\left(\Pi^{+}\right) \rightarrow \mathcal{B}^{\alpha}\left(\Pi^{+}\right)$}

In this section, we characterize the boundedness and compactness of the operator $T_{\psi_{1}, \psi_{2}, \varphi}: H^{p}\left(\Pi^{+}\right) \rightarrow \mathcal{B}^{\alpha}\left(\Pi^{+}\right)$. We also give upper and lower bounds for the norm of $T_{\psi_{1}, \psi_{2}, \varphi}$ acting between these spaces.

Theorem 4 Let $1 \leq p<\infty, \alpha>0, \psi_{1}, \psi_{2} \in H\left(\Pi^{+}\right)$and $\varphi \in S\left(\Pi^{+}\right)$. Then $T_{\psi_{1}, \psi_{2}, \varphi}$ : $H^{p}\left(\Pi^{+}\right) \rightarrow \mathcal{B}^{\alpha}\left(\Pi^{+}\right)$is bounded if and only if the following conditions are satisfied:

(i) $\quad M_{1}=\sup _{z \in \Pi^{+}} \frac{(\Im z)^{\alpha}}{(\Im \varphi(z))^{1 / p}}\left|\psi_{1}^{\prime}(z)\right|<\infty$;

(ii) $\quad M_{2}=\sup _{z \in \Pi^{+}} \frac{(\Im z)^{\alpha}}{(\Im \varphi(z))^{1+1 / p}}\left|\psi_{1}(z) \varphi^{\prime}(z)+\psi_{2}^{\prime}(z)\right|<\infty$;

(iii) $\quad M_{3}=\sup _{z \in \Pi^{+}} \frac{(\Im z)^{\alpha}}{(\Im \varphi(z))^{2+1 / p}}\left|\psi_{2}(z) \varphi^{\prime}(z)\right|<\infty$.

Moreover,

$$
\begin{aligned}
M_{1}+M_{2}+M_{3} & \lesssim\left\|T_{\psi_{1}, \psi_{2}, \varphi}\right\|_{H^{p}\left(\Pi^{+}\right) \rightarrow \mathcal{B}^{\alpha}\left(\Pi^{+}\right)} \\
& \lesssim \frac{\left|\psi_{1}(i)\right|}{(\Im \varphi(i))^{1 / p}}+\frac{\left|\psi_{2}(i)\right|}{(\Im \varphi(i))^{1+1 / p}}+M_{1}+M_{2}+M_{3} .
\end{aligned}
$$

Proof First, suppose that conditions (i)-(iii) hold. Then by Lemma 1 we have

$$
\begin{aligned}
(\Im z)^{\alpha}\left|\left(T_{\psi_{1}, \psi_{2}, \varphi} f\right)^{\prime}(z)\right| & \\
= & (\Im z)^{\alpha}\left|\psi_{1}^{\prime}(z) f(\varphi(z))+\left(\psi_{1}(z) \varphi^{\prime}(z)+\psi_{2}^{\prime}(z)\right) f^{\prime}(\varphi(z))+\psi_{2}(z) \varphi^{\prime}(z) f^{\prime \prime}(\varphi(z))\right| \\
\leq & C\|f\|_{H^{p}\left(\Pi^{+}\right)}\left(\frac{(\Im z)^{\alpha}}{(\Im \varphi(z))^{1 / p}}\left|\psi_{1}^{\prime}(z)\right|+\frac{(\Im z)^{\alpha}}{(\Im \varphi(z))^{1+1 / p}}\left|\psi_{1}(z) \varphi^{\prime}(z)+\psi_{2}^{\prime}(z)\right|\right. \\
& \left.+\frac{(\Im z)^{\alpha}}{(\Im \varphi(z))^{2+1 / p}}\left|\psi_{2}(z) \varphi^{\prime}(z)\right|\right) \\
\leq & C\left(M_{1}+M_{2}+M_{3}\right)\|f\|_{H^{p}\left(\Pi^{+}\right)} .
\end{aligned}
$$

We also have

$$
\begin{aligned}
\left|T_{\psi_{1}, \psi_{2}, \varphi} f(i)\right| & =\left|\psi_{1}(i) f(\varphi(i))+\psi_{2}(i) f^{\prime}(\varphi(i))\right| \\
& \leq C\|f\|_{H^{p}\left(\Pi^{+}\right)}\left(\frac{\left|\psi_{1}(i)\right|}{(\Im \varphi(i))^{1 / p}}+\frac{\left|\psi_{2}(i)\right|}{(\Im \varphi(i))^{1+1 / p}}\right) .
\end{aligned}
$$


Combining (19) and (20), we have

$$
\left\|T_{\psi_{1}, \psi_{2}, \varphi} f\right\|_{\mathcal{B}^{\alpha}\left(\Pi^{+}\right)} \lesssim\left(\frac{\left|\psi_{1}(i)\right|}{(\Im \varphi(i))^{1 / p}}+\frac{\left|\psi_{2}(i)\right|}{(\Im \varphi(i))^{1+1 / p}}+M_{1}+M_{2}+M_{3}\right)\|f\|_{H^{p}\left(\Pi^{+}\right)},
$$

from which it follows that

$$
\left\|T_{\psi_{1}, \psi_{2}, \varphi}\right\|_{H^{p}\left(\Pi^{+}\right) \rightarrow \mathcal{B}^{\alpha}\left(\Pi^{+}\right)} \lesssim \frac{\left|\psi_{1}(i)\right|}{(\Im \varphi(i))^{1 / p}}+\frac{\left|\psi_{2}(i)\right|}{(\Im \varphi(i))^{1+1 / p}}+M_{1}+M_{2}+M_{3} .
$$

Conversely, suppose that $T_{\psi_{1}, \psi_{2}, \varphi}: H^{p}\left(\Pi^{+}\right) \rightarrow \mathcal{B}^{\alpha}\left(\Pi^{+}\right)$is bounded. Consider the family of functions

$$
f_{w}(z)=\frac{(\Im w)^{2-1 / p}}{\pi^{1 / p}(z-\bar{w})^{2}}-4 i \frac{(\Im w)^{3-1 / p}}{\pi^{1 / p}(z-\bar{w})^{3}}-4 \frac{(\Im w)^{4-1 / p}}{\pi^{1 / p}(z-\bar{w})^{4}}
$$

where $w \in \Pi^{+}$.

Since the functions $f_{w}$ are linear combinations of the functions in Lemma 2 (for $k=$ $2,3,4$ ), from this by using the lemma it follows that

$$
L_{1}:=\sup _{w \in \Pi^{+}}\left\|f_{w}\right\|_{H^{p}\left(\Pi^{+}\right)}<\infty .
$$

We also have

$$
\begin{aligned}
& f_{w}^{\prime}(z)=\frac{-2(\Im w)^{2-1 / p}}{\pi^{1 / p}(z-\bar{w})^{3}}+12 i \frac{(\Im w)^{3-1 / p}}{\pi^{1 / p}(z-\bar{w})^{4}}+16 \frac{(\Im w)^{4-1 / p}}{\pi^{1 / p}(z-\bar{w})^{5}}, \\
& f_{w}^{\prime \prime}(z)=\frac{6(\Im w)^{2-1 / p}}{\pi^{1 / p}(z-\bar{w})^{4}}-48 i \frac{(\Im w)^{3-1 / p}}{\pi^{1 / p}(z-\bar{w})^{5}}-80 \frac{(\Im w)^{4-1 / p}}{\pi^{1 / p}(z-\bar{w})^{6}},
\end{aligned}
$$

from which with some simple calculation we obtain

$$
f_{w}(w)=0, \quad f_{w}^{\prime}(w)=0 \quad \text { and } \quad f_{w}^{\prime \prime}(w)=\frac{1}{8 \pi^{1 / p}(\Im w)^{2+1 / p}} .
$$

Since $T_{\psi_{1}, \psi_{2}, \varphi}: H^{p}\left(\Pi^{+}\right) \rightarrow \mathcal{B}^{\alpha}\left(\Pi^{+}\right)$is bounded, we have

$$
\begin{aligned}
\left\|T_{\psi_{1}, \psi_{2}, \varphi} f_{w}\right\|_{\mathcal{B}^{\alpha}\left(\Pi^{+}\right)} & \leq\left\|T_{\psi_{1}, \psi_{2}, \varphi}\right\|_{H^{p}\left(\Pi^{+}\right) \rightarrow \mathcal{B}^{\alpha}\left(\Pi^{+}\right)}\left\|f_{w}\right\|_{H^{p}\left(\Pi^{+}\right)} \\
& \leq L_{1}\left\|T_{\psi_{1}, \psi_{2}, \varphi}\right\|_{H^{p}\left(\Pi^{+}\right) \rightarrow \mathcal{B}^{\alpha}\left(\Pi^{+}\right)}
\end{aligned}
$$

for every $w \in \Pi^{+}$.

Thus for each $\zeta \in \Pi^{+}$, we have

$$
\begin{aligned}
L_{1}\left\|T_{\psi_{1}, \psi_{2}, \varphi}\right\|_{H^{p}\left(\Pi^{+}\right) \rightarrow \mathcal{B}^{\alpha}\left(\Pi^{+}\right)} \geq & (\Im \zeta)^{\alpha}\left|\left(T_{\psi_{1}, \psi_{2}, \varphi} f_{\varphi(\zeta)}\right)^{\prime}(\zeta)\right| \\
= & (\Im \zeta)^{\alpha} \mid \psi_{1}^{\prime}(\zeta) f_{\varphi(\zeta)}(\varphi(\zeta))+\left(\psi_{1}(\zeta) \varphi^{\prime}(\zeta)+\psi_{2}^{\prime}(\zeta)\right) f_{\varphi(\zeta)}^{\prime}(\varphi(\zeta)) \\
& +\psi_{2}(\zeta) \varphi^{\prime}(\zeta) f_{\varphi(\zeta)}^{\prime \prime}(\varphi(\zeta)) \mid \\
= & \frac{(\Im \zeta)^{\alpha}\left|\psi_{2}(\zeta) \varphi^{\prime}(\zeta)\right|}{8 \pi^{1 / p}(\Im \varphi(\zeta))^{2+1 / p}} .
\end{aligned}
$$


Since $\zeta \in \Pi^{+}$is arbitrary, we have that

$$
M_{3}=\sup _{\zeta \in \Pi^{+}} \frac{(\Im \zeta)^{\alpha}\left|\psi_{2}(\zeta) \varphi^{\prime}(\zeta)\right|}{(\Im \varphi(\zeta))^{2+1 / p}} \leq 8 \pi^{1 / p} L_{1}\left\|T_{\psi_{1}, \psi_{2}, \varphi}\right\|_{H^{p}\left(\Pi^{+}\right) \rightarrow \mathcal{B}^{\alpha}\left(\Pi^{+}\right)}
$$

Now, consider the family of functions

$$
g_{w}(z)=12 \frac{(\Im w)^{2-1 / p}}{\pi^{1 / p}(z-\bar{w})^{2}}-32 i \frac{(\Im w)^{3-1 / p}}{\pi^{1 / p}(z-\bar{w})^{3}}-24 \frac{(\Im w)^{4-1 / p}}{\pi^{1 / p}(z-\bar{w})^{4}}
$$

Since the functions $g_{w}$ are also linear combinations of the functions in Lemma 2, we have $L_{2}:=\sup _{w \in \Pi^{+}}\left\|g_{w}\right\|_{H^{p}\left(\Pi^{+}\right)} \leq 1$.

We also have

$$
\begin{aligned}
& g_{w}^{\prime}(z)=-24 \frac{(\Im w)^{2-1 / p}}{\pi^{1 / p}(z-\bar{w})^{3}}+96 i \frac{(\Im w)^{3-1 / p}}{\pi^{1 / p}(z-\bar{w})^{4}}+96 \frac{(\Im w)^{4-1 / p}}{\pi^{1 / p}(z-\bar{w})^{5}}, \\
& g_{w}^{\prime \prime}(z)=72 \frac{(\Im w)^{2-1 / p}}{\pi^{1 / p}(z-\bar{w})^{4}}-384 i \frac{(\Im w)^{3-1 / p}}{\pi^{1 / p}(z-\bar{w})^{5}}-480 \frac{(\Im w)^{4-1 / p}}{\pi^{1 / p}(z-\bar{w})^{6}},
\end{aligned}
$$

from which it follows that

$$
g_{w}^{\prime}(w)=0, \quad g_{w}^{\prime \prime}(w)=0, \quad \text { and } \quad g_{w}(w)=-\frac{1}{2 \pi^{1 / p}(\Im w)^{1 / p}}
$$

Since $T_{\psi_{1}, \psi_{2}, \varphi}: H^{p}\left(\Pi^{+}\right) \rightarrow \mathcal{B}^{\alpha}\left(\Pi^{+}\right)$is bounded, for each $\zeta \in \Pi^{+}$, we have

$$
\begin{aligned}
L_{2}\left\|T_{\psi_{1}, \psi_{2}, \varphi}\right\|_{H^{p}\left(\Pi^{+}\right) \rightarrow \mathcal{B}^{\alpha}\left(\Pi^{+}\right) \geq} & \left\|T_{\psi_{1}, \psi_{2}, \varphi} g_{\varphi(\zeta)}\right\|_{\mathcal{B}^{\alpha}\left(\Pi^{+}\right)} \\
= & (\Im \zeta)^{\alpha} \mid \psi_{1}^{\prime}(\zeta) g_{\varphi(\zeta)}(\varphi(\zeta))+\left(\psi_{1}(\zeta) \varphi^{\prime}(\zeta)+\psi_{2}^{\prime}(\zeta)\right) g_{\varphi(\zeta)}^{\prime}(\varphi(\zeta)) \\
& +\psi_{2}(\zeta) \varphi^{\prime}(\zeta) g_{\varphi(\zeta)}^{\prime \prime}(\varphi(\zeta)) \mid \\
= & \frac{(\Im \zeta)^{\alpha}\left|\psi_{1}^{\prime}(\zeta)\right|}{2 \pi^{1 / p}(\Im \varphi(\zeta))^{1 / p}} .
\end{aligned}
$$

Since $\zeta \in \Pi^{+}$is arbitrary, we have that

$$
M_{1}=\sup _{\zeta \in \Pi^{+}} \frac{(\Im \zeta)^{\alpha}\left|\psi_{1}^{\prime}(\zeta)\right|}{(\Im \varphi(\zeta))^{1 / p}} \leq 2 \pi^{1 / p} L_{2}\left\|T_{\psi_{1}, \psi_{2}, \varphi}\right\|_{H^{p}\left(\Pi^{+}\right) \rightarrow \mathcal{B}^{\alpha}\left(\Pi^{+}\right)}
$$

Now, consider the family of functions

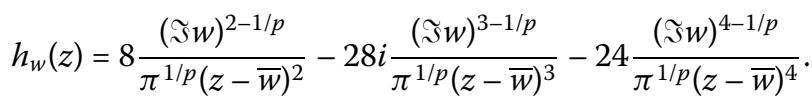

Once again proceeding as before, we can show that $\sup _{w \in \Pi^{+}}\left\|h_{w}\right\|_{H^{p}\left(\Pi^{+}\right)} \lesssim 1$.

We have

$$
\begin{aligned}
& h_{w}^{\prime}(z)=-16 \frac{(\Im w)^{2-1 / p}}{\pi^{1 / p}(z-\bar{w})^{3}}+84 i \frac{(\Im w)^{3-1 / p}}{\pi^{1 / p}(z-\bar{w})^{4}}+96 \frac{(\Im w)^{4-1 / p}}{\pi^{1 / p}(z-\bar{w})^{5}} \\
& h_{w}^{\prime \prime}(z)=48 \frac{(\Im w)^{2-1 / p}}{\pi^{1 / p}(z-\bar{w})^{4}}-336 i \frac{(\Im w)^{3-1 / p}}{\pi^{1 / p}(z-\bar{w})^{5}}-480 \frac{(\Im w)^{4-1 / p}}{\pi^{1 / p}(z-\bar{w})^{6}} .
\end{aligned}
$$


Thus

$$
h_{w}(w)=0, \quad h_{w}^{\prime \prime}(w)=0 \quad \text { and } \quad h_{w}^{\prime}(w)=\frac{i}{4 \pi^{1 / p}(\Im w)^{1+1 / p}} .
$$

Therefore by the boundedness of $T_{\psi_{1}, \psi_{2}, \varphi}: H^{p}\left(\Pi^{+}\right) \rightarrow \mathcal{B}^{\alpha}\left(\Pi^{+}\right)$, for each $\zeta \in \Pi^{+}$, we have

$$
\begin{aligned}
L_{3}\left\|T_{\psi_{1}, \psi_{2}, \varphi}\right\|_{H^{p}\left(\Pi^{+}\right) \rightarrow \mathcal{B}^{\alpha}\left(\Pi^{+}\right)} \geq & (\Im \zeta)^{\alpha} \mid \psi_{1}^{\prime}(\zeta) h_{\varphi(\zeta)}(\varphi(\zeta))+\left(\psi_{1}(\zeta) \varphi^{\prime}(\zeta)+\psi_{2}^{\prime}(\zeta)\right) h_{\varphi(\zeta)}^{\prime}(\varphi(\zeta)) \\
& +\psi_{2}(\zeta) \varphi^{\prime}(\zeta) h_{\varphi(\zeta)}^{\prime \prime}(\varphi(\zeta)) \mid \\
= & \frac{(\Im \zeta)^{\alpha}\left|\psi_{1}(\zeta) \varphi^{\prime}(\zeta)+\psi_{2}^{\prime}(\zeta)\right|}{4 \pi^{1 / p}(\Im \varphi(\zeta))^{1+1 / p}}
\end{aligned}
$$

and consequently

$$
M_{2}=\sup _{\zeta \in \Pi^{+}} \frac{(\Im \zeta)^{\alpha}\left|\psi_{1}(\zeta) \varphi^{\prime}(\zeta)+\psi_{2}^{\prime}(\zeta)\right|}{(\Im \varphi(\zeta))^{1+1 / p}} \leq 4 \pi^{1 / p} L_{3}\left\|T_{\psi_{1}, \psi_{2}, \varphi}\right\|_{H^{p}\left(\Pi^{+}\right) \rightarrow \mathcal{B}^{\alpha}\left(\Pi^{+}\right)} .
$$

Combining (24), (25), and (26), we have

$$
M_{1}+M_{2}+M_{3} \lesssim\left\|T_{\psi_{1}, \psi_{2}, \varphi}\right\|_{H^{p}\left(\Pi^{+}\right) \rightarrow \mathcal{B}^{\alpha}\left(\Pi^{+}\right)}
$$

finishing the proof of the theorem.

Corollary 5 Let $1 \leq p<\infty, \alpha>0$ and $\varphi \in S\left(\Pi^{+}\right)$. Then $C_{\varphi}: H^{p}\left(\Pi^{+}\right) \rightarrow \mathcal{B}^{\alpha}\left(\Pi^{+}\right)$is bounded if and only if

$$
M_{4}=\sup _{z \in \Pi^{+}} \frac{(\Im z)^{\alpha}}{(\Im \varphi(z))^{1+1 / p}}\left|\varphi^{\prime}(z)\right|<\infty .
$$

Moreover,

$$
M_{4} \lesssim\left\|C_{\varphi}\right\|_{H^{p}\left(\Pi^{+}\right) \rightarrow \mathcal{B}^{\alpha}\left(\Pi^{+}\right)} \lesssim \frac{1}{(\Im \varphi(i))^{1 / p}}+M_{4} .
$$

Corollary 6 Let $1 \leq p<\infty, \alpha>0, \psi \in H\left(\Pi^{+}\right)$and $\varphi \in S\left(\Pi^{+}\right)$. Then $M_{\psi} C_{\varphi}: H^{p}\left(\Pi^{+}\right) \rightarrow$ $\mathcal{B}^{\alpha}\left(\Pi^{+}\right)$is bounded if and only if

(i) $\quad M_{5}=\sup _{z \in \Pi^{+}} \frac{(\Im z)^{\alpha}}{(\Im \varphi(z))^{1 / p}}\left|\psi^{\prime}(z)\right|<\infty$,

(ii) $M_{6}=\sup _{z \in \Pi^{+}} \frac{(\Im z)^{\alpha}}{(\Im \varphi(z))^{1+1 / p}}\left|\psi(z) \varphi^{\prime}(z)\right|<\infty$.

Moreover,

$$
M_{5}+M_{6} \lesssim\left\|M_{\psi} C_{\varphi}\right\|_{H^{p}\left(\Pi^{+}\right) \rightarrow \mathcal{B}^{\alpha}\left(\Pi^{+}\right)} \lesssim \frac{|\psi(i)|}{(\Im \varphi(i))^{1 / p}}+M_{5}+M_{6} .
$$

Corollary 7 Let $1 \leq p<\infty, \alpha>0, \psi \in H\left(\Pi^{+}\right)$and $\varphi \in S\left(\Pi^{+}\right)$. Then $C_{\varphi} M_{\psi}: H^{p}\left(\Pi^{+}\right) \rightarrow$ $\mathcal{B}^{\alpha}\left(\Pi^{+}\right)$is bounded if and only if

(i) $\quad M_{7}=\sup _{z \in \Pi^{+}} \frac{(\Im z)^{\alpha}}{(\Im \varphi(z))^{1 / p}}\left|\psi^{\prime}(\varphi(z)) \varphi^{\prime}(z)\right|<\infty$,

(ii) $\quad M_{8}=\sup _{z \in \Pi^{+}} \frac{(\Im z)^{\alpha}}{(\Im \varphi(z))^{1+1 / p}}\left|\psi(\varphi(z)) \varphi^{\prime}(z)\right|<\infty$. 
Moreover,

$$
M_{7}+M_{8} \lesssim\left\|C_{\varphi} M_{\psi}\right\|_{H^{p}\left(\Pi^{+}\right) \rightarrow \mathcal{B}^{\alpha}\left(\Pi^{+}\right)} \lesssim \frac{|\psi(\varphi(i))|}{(\Im \varphi(i))^{1 / p}}+M_{7}+M_{8}
$$

Corollary 8 Let $1 \leq p<\infty, \alpha>0$ and $\varphi \in S\left(\Pi^{+}\right)$. Then $C_{\varphi} D: H^{p}\left(\Pi^{+}\right) \rightarrow \mathcal{B}^{\alpha}\left(\Pi^{+}\right)$is bounded if and only if

$$
M_{9}=\sup _{z \in \Pi^{+}} \frac{(\Im z)^{\alpha}}{(\Im \varphi(z))^{2+1 / p}}\left|\varphi^{\prime}(z)\right|<\infty .
$$

Moreover,

$$
M_{9} \lesssim\left\|C_{\varphi} D\right\|_{H^{p}\left(\Pi^{+}\right) \rightarrow \mathcal{B}^{\alpha}\left(\Pi^{+}\right)} \lesssim \frac{1}{(\Im \varphi(i))^{1+1 / p}}+M_{9}
$$

Corollary 9 Let $1 \leq p<\infty, \alpha>0$ and $\varphi \in S\left(\Pi^{+}\right)$. Then $D C_{\varphi}: H^{p}\left(\Pi^{+}\right) \rightarrow \mathcal{B}^{\alpha}\left(\Pi^{+}\right)$is bounded if and only if

(i) $\quad M_{10}=\sup _{z \in \Pi^{+}} \frac{(\Im z)^{\alpha}}{(\Im \varphi(z))^{1+1 / p}}\left|\varphi^{\prime \prime}(z)\right|<\infty$,

(ii) $\quad M_{11}=\sup _{z \in \Pi^{+}} \frac{(\Im z)^{\alpha}}{(\Im \varphi(z))^{2+1 / p}}\left|\varphi^{\prime}(z)\right|^{2}<\infty$.

Moreover,

$$
M_{10}+M_{11} \lesssim\left\|D C_{\varphi}\right\|_{H^{p}\left(\Pi^{+}\right) \rightarrow \mathcal{B}^{\alpha}\left(\Pi^{+}\right)} \lesssim \frac{\left|\varphi^{\prime}(i)\right|}{(\Im \varphi(i))^{1+1 / p}}+M_{10}+M_{11}
$$

Corollary 10 Let $1 \leq p<\infty, \alpha>0, \psi \in H\left(\Pi^{+}\right)$and $\varphi \in S\left(\Pi^{+}\right)$. Then $M_{\psi} C_{\varphi} D: H^{p}\left(\Pi^{+}\right) \rightarrow$ $\mathcal{B}^{\alpha}\left(\Pi^{+}\right)$is bounded if and only if

(i) $\quad M_{12}=\sup _{z \in \Pi^{+}} \frac{(\Im z)^{\alpha}}{(\Im \varphi(z))^{1+1 / p}}\left|\psi^{\prime}(z)\right|<\infty$,

(ii) $\quad M_{13}=\sup _{z \in \Pi^{+}} \frac{(\Im z)^{\alpha}}{(\Im \varphi(z))^{2+1 / p}}\left|\psi(z) \varphi^{\prime}(z)\right|<\infty$.

Moreover,

$$
M_{12}+M_{13} \lesssim\left\|M_{\psi} C_{\varphi} D\right\|_{H^{p}\left(\Pi^{+}\right) \rightarrow \mathcal{B}^{\alpha}\left(\Pi^{+}\right)} \lesssim \frac{|\psi(i)|}{(\Im \varphi(i))^{1+1 / p}}+M_{12}+M_{13}
$$

Corollary 11 Let $1 \leq p<\infty, \alpha>0, \psi \in H\left(\Pi^{+}\right)$and $\varphi \in S\left(\Pi^{+}\right)$. Then $C_{\varphi} M_{\psi} D: H^{p}\left(\Pi^{+}\right) \rightarrow$ $\mathcal{B}^{\alpha}\left(\Pi^{+}\right)$is bounded if and only if

(i) $\quad M_{14}=\sup _{z \in \Pi^{+}} \frac{(\Im z)^{\alpha}}{(\Im \varphi(z))^{1+1 / p}}\left|\psi^{\prime}(\varphi(z)) \varphi^{\prime}(z)\right|<\infty$,

(ii) $\quad M_{15}=\sup _{z \in \Pi^{+}} \frac{(\Im z)^{\alpha}}{(\Im \varphi(z))^{2+1 / p}}\left|\psi(\varphi(z)) \varphi^{\prime}(z)\right|<\infty$. 
Moreover,

$$
M_{14}+M_{15} \lesssim\left\|C_{\varphi} M_{\psi} D\right\|_{H^{p}\left(\Pi^{+}\right) \rightarrow \mathcal{B}^{\alpha}\left(\Pi^{+}\right)} \lesssim \frac{|\psi(\varphi(i))|}{(\Im \varphi(i))^{1+1 / p}}+M_{14}+M_{15}
$$

Corollary 12 Let $1 \leq p<\infty, \alpha>0, \psi \in H\left(\Pi^{+}\right)$and $\varphi \in S\left(\Pi^{+}\right)$. Then $M_{\psi} D C_{\varphi}: H^{p}\left(\Pi^{+}\right) \rightarrow$ $\mathcal{B}^{\alpha}\left(\Pi^{+}\right)$is bounded if and only if

(i) $\quad M_{16}=\sup _{z \in \Pi^{+}} \frac{(\Im z)^{\alpha}}{(\Im \varphi(z))^{1+1 / p}}\left|\left(\psi \varphi^{\prime}\right)^{\prime}(z)\right|<\infty$,

(ii) $\quad M_{17}=\sup _{z \in \Pi^{+}} \frac{(\Im z)^{\alpha}}{(\Im \varphi(z))^{2+1 / p}}\left|\psi(z)\left(\varphi^{\prime}(z)\right)^{2}\right|<\infty$.

Moreover,

$$
M_{16}+M_{17} \lesssim\left\|M_{\psi} D C_{\varphi}\right\|_{H^{p}\left(\Pi^{+}\right) \rightarrow \mathcal{B}^{\alpha}\left(\Pi^{+}\right)} \lesssim \frac{\left|\psi(i) \varphi^{\prime}(i)\right|}{(\Im \varphi(i))^{1+1 / p}}+M_{16}+M_{17}
$$

Corollary 13 Let $1 \leq p<\infty, \alpha>0, \psi \in H\left(\Pi^{+}\right)$and $\varphi \in S\left(\Pi^{+}\right)$. Then $D M_{\psi} C_{\varphi}: H^{p}\left(\Pi^{+}\right) \rightarrow$ $\mathcal{B}^{\alpha}\left(\Pi^{+}\right)$is bounded if and only if

(i) $\quad M_{18}=\sup _{z \in \Pi^{+}} \frac{(\Im z)^{\alpha}}{(\Im \varphi(z))^{1 / p}}\left|\psi^{\prime \prime}(z)\right|<\infty$,

(ii) $\quad M_{19}=\sup _{z \in \Pi^{+}} \frac{(\Im z)^{\alpha}}{(\Im \varphi(z))^{1+1 / p}}\left|2 \psi^{\prime}(z) \varphi^{\prime}(z)+\psi(z) \varphi^{\prime \prime}(z)\right|<\infty$,

(iii) $\quad M_{20}=\sup _{z \in \Pi^{+}} \frac{(\Im z)^{\alpha}}{(\Im \varphi(z))^{2+1 / p}}\left|\psi(z)\left(\varphi^{\prime}(z)\right)^{2}\right|<\infty$.

Moreover,

$$
\begin{aligned}
M_{18}+M_{19}+M_{20} & \lesssim\left\|D M_{\psi} C_{\varphi}\right\|_{H^{p}\left(\Pi^{+}\right) \rightarrow \mathcal{B}^{\alpha}\left(\Pi^{+}\right)} \\
& \lesssim \frac{\left|\psi^{\prime}(i)\right|}{(\Im \varphi(i))^{1 / p}}+\frac{\left|\psi(i) \varphi^{\prime}(i)\right|}{(\Im \varphi(i))^{1+1 / p}}+M_{18}+M_{19}+M_{20} .
\end{aligned}
$$

Corollary 14 Let $1 \leq p<\infty, \alpha>0, \psi \in H\left(\Pi^{+}\right)$and $\varphi \in S\left(\Pi^{+}\right)$. Then $C_{\varphi} D M_{\psi}: H^{p}\left(\Pi^{+}\right) \rightarrow$ $\mathcal{B}^{\alpha}\left(\Pi^{+}\right)$is bounded if and only if
(i) $\quad M_{21}=\sup _{z \in \Pi^{+}} \frac{(\Im z)^{\alpha}}{(\Im \varphi(z))^{1 / p}}\left|\psi^{\prime \prime}(\varphi(z)) \varphi^{\prime}(z)\right|<\infty$,
(ii) $\quad M_{22}=\sup _{z \in \Pi^{+}} \frac{(\Im z)^{\alpha}}{(\Im \varphi(z))^{1+1 / p}}\left|\psi^{\prime}(\varphi(z)) \varphi^{\prime}(z)\right|<\infty$,
(iii) $\quad M_{23}=\sup _{z \in \Pi^{+}} \frac{(\Im z)^{\alpha}}{(\Im \varphi(z))^{2+1 / p}}\left|\psi(\varphi(z)) \varphi^{\prime}(z)\right|<\infty$.

Moreover,

$$
\begin{aligned}
M_{21}+M_{22}+M_{23} & \lesssim\left\|C_{\varphi} D M_{\psi}\right\|_{H^{p}\left(\Pi^{+}\right) \rightarrow \mathcal{B}^{\alpha}\left(\Pi^{+}\right)} \\
& \lesssim \frac{\left|\psi^{\prime}(\varphi(i))\right|}{(\Im \varphi(i))^{1 / p}}+\frac{|\psi(\varphi(i))|}{(\Im \varphi(i))^{1+1 / p}}+M_{21}+M_{22}+M_{23} .
\end{aligned}
$$


Corollary 15 Let $1 \leq p<\infty, \alpha>0, \psi \in H\left(\Pi^{+}\right)$and $\varphi \in S\left(\Pi^{+}\right)$. Then $D C_{\varphi} M_{\psi}: H^{p}\left(\Pi^{+}\right) \rightarrow$ $\mathcal{B}^{\alpha}\left(\Pi^{+}\right)$is bounded if and only if

(i) $\quad M_{24}=\sup _{z \in \Pi^{+}} \frac{(\Im z)^{\alpha}}{(\Im \varphi(z))^{1 / p}}\left|\psi^{\prime}(\varphi(z)) \varphi^{\prime \prime}(z)+\psi^{\prime \prime}(\varphi(z))\left(\varphi^{\prime}(z)\right)^{2}\right|<\infty$,

(ii) $\quad M_{25}=\sup _{z \in \Pi^{+}} \frac{(\Im z)^{\alpha}}{(\Im \varphi(z))^{1+1 / p}}\left|2 \psi^{\prime}(\varphi(z))\left(\varphi^{\prime}(z)\right)^{2}+\psi(\varphi(z)) \varphi^{\prime \prime}(z)\right|<\infty$,

(iii) $\quad M_{26}=\sup _{z \in \Pi^{+}} \frac{(\Im z)^{\alpha}}{(\Im \varphi(z))^{2+1 / p}}\left|\psi(\varphi(z))\left(\varphi^{\prime}(z)\right)^{2}\right|<\infty$.

Moreover,

$$
\begin{aligned}
M_{24}+M_{25}+M_{26} & \lesssim\left\|D C_{\varphi} M_{\psi}\right\|_{H^{p}\left(\Pi^{+}\right) \rightarrow \mathcal{B}^{\alpha}\left(\Pi^{+}\right)} \\
& \lesssim \frac{\left|\psi^{\prime}(\varphi(i)) \varphi^{\prime}(i)\right|}{(\Im \varphi(i))^{1 / p}}+\frac{\left|\psi(\varphi(i)) \varphi^{\prime}(i)\right|}{(\Im \varphi(i))^{1+1 / p}}+M_{24}+M_{25}+M_{26} .
\end{aligned}
$$

Recall that for a function $f$ defined in $\Pi^{+}, \lim _{z \rightarrow \partial \widehat{\Pi^{+}}} f(z)=0$ if and only if for every $\varepsilon>0$, there is a compact set $K \subset \Pi^{+}$such that $|f(z)|<\varepsilon$ for $z \in \Pi^{+} \backslash K$.

The following result characterizes the compactness of the operator $T_{\psi_{1}, \psi_{2}, \varphi}: H^{p}\left(\Pi^{+}\right) \rightarrow$ $\mathcal{B}^{\alpha}\left(\Pi^{+}\right)$.

Theorem 16 Let $1 \leq p<\infty, \alpha>0, \psi_{1}, \psi_{2} \in H\left(\Pi^{+}\right)$, and $\varphi \in S\left(\Pi^{+}\right)$. Then $T_{\psi_{1}, \psi_{2}, \varphi}$ : $H^{p}\left(\Pi^{+}\right) \rightarrow \mathcal{B}^{\alpha}\left(\Pi^{+}\right)$is compact if and only if it is bounded and the following conditions are satisfied:

$$
\begin{aligned}
& \text { (i) } \lim _{\varphi(z) \rightarrow \partial \widetilde{\Pi}^{+}} \frac{(\Im z)^{\alpha}}{(\Im \varphi(z))^{1 / p}}\left|\psi_{1}^{\prime}(z)\right|=0, \\
& \text { (ii) } \lim _{\varphi(z) \rightarrow \partial \widetilde{\Pi}^{+}} \frac{(\Im z)^{\alpha}}{(\Im \varphi(z))^{1+1 / p}}\left|\psi_{1}(z) \varphi^{\prime}(z)+\psi_{2}^{\prime}(z)\right|=0, \\
& \text { (iii) } \quad \lim _{\varphi(z) \rightarrow \partial \widetilde{\Pi}^{+}} \frac{(\Im z)^{\alpha}}{(\Im \varphi(z))^{2+1 / p}}\left|\psi_{2}(z) \varphi^{\prime}(z)\right|=0 .
\end{aligned}
$$

Proof Assume that the operator is bounded and conditions (i)-(iii) hold. Then by Theorem 4 the quantities $M_{j}, j=\overline{1,3}$, are finite, whereas from conditions (i)-(iii) we have that, for each $\varepsilon>0$, there exists a compact set $K$ in $\Pi^{+}$such that

$$
\begin{aligned}
& \frac{(\Im z)^{\alpha}}{(\Im \varphi(z))^{1 / p}}\left|\psi_{1}^{\prime}(z)\right|<\varepsilon, \\
& \frac{(\Im z)^{\alpha}}{(\Im \varphi(z))^{1+1 / p}}\left|\psi_{1}(z) \varphi^{\prime}(z)+\psi_{2}^{\prime}(z)\right|<\varepsilon, \\
& \frac{(\Im z)^{\alpha}}{(\Im \varphi(z))^{2+1 / p}}\left|\psi_{2}(z) \varphi^{\prime}(z)\right|<\varepsilon,
\end{aligned}
$$

provided that $\varphi(z) \in \Pi^{+} \backslash K$.

Let $\left(f_{n}\right)_{n \in \mathbb{N}}$ be a sequence in $H^{p}\left(\Pi^{+}\right)$weakly convergent to zero. Then, by inequalities (35)-(37) and Lemma 1, for every $z \in \Pi^{+}$with $\varphi(z) \in \Pi^{+} \backslash K$, we have 


$$
\begin{aligned}
(\Im z)^{\alpha} & \left|\left(T_{\psi_{1}, \psi_{2}, \varphi} f_{n}\right)^{\prime}(z)\right| \\
\leq & (\Im z)^{\alpha}\left|\psi_{1}^{\prime}(z) f_{n}(\varphi(z))+\left(\psi_{1}(z) \varphi^{\prime}(z)+\psi_{2}^{\prime}(z)\right) f_{n}^{\prime}(\varphi(z))+\psi_{2}(z) \varphi^{\prime}(z) f_{n}^{\prime \prime}(\varphi(z))\right| \\
\leq & C\left(\frac{(\Im z)^{\alpha}}{(\Im \varphi(z))^{1 / p}}\left|\psi_{1}^{\prime}(z)\right|+\frac{(\Im z)^{\alpha}}{(\Im \varphi(z))^{1+1 / p}}\left|\psi_{1}(z) \varphi^{\prime}(z)+\psi_{2}^{\prime}(z)\right|\right. \\
& \left.+\frac{(\Im z)^{\alpha}}{(\Im \varphi(z))^{2+1 / p}}\left|\psi_{2}(z) \varphi^{\prime}(z)\right|\right)\left\|f_{n}\right\|_{H^{p}\left(\Pi^{+}\right)} \\
< & C \varepsilon .
\end{aligned}
$$

Since $K$ is compact, $\varphi(K)$ is also compact. Hence

$$
M_{27}:=\sup _{w \in \varphi(z)} \Im w \in(0, \infty)
$$

Choose $n_{0} \in \mathbb{N}$ such that

$$
\begin{aligned}
& \max _{z \in K}\left|f_{n}(z)\right|<\frac{\varepsilon}{M_{1} M_{27}^{1 / p}}, \quad \max _{z \in K}\left|f_{n}^{\prime}(z)\right|<\frac{\varepsilon}{M_{2} M_{27}^{1 / p+1}}, \\
& \max _{z \in K}\left|f_{n}^{\prime \prime}(z)\right|<\frac{\varepsilon}{M_{3} M_{27}^{1 / p+2}},
\end{aligned}
$$

and

$$
\max \left\{\left|f_{n}(i)\right|,\left|f_{n}^{\prime}(i)\right|\right\}<\varepsilon
$$

for all $n \geq n_{0}$.

Then, by the preceding and (38), for every $z \in \Pi^{+}$such that $\varphi(z) \in K$, we have

$$
\begin{aligned}
(\Im z)^{\alpha} & \left|\psi_{1}^{\prime}(z) f_{n}(\varphi(z))+\left(\psi_{1}(z) \varphi^{\prime}(z)+\psi_{2}^{\prime}(z)\right) f_{n}^{\prime}(\varphi(z))+\psi_{2}(z) \varphi^{\prime}(z) f_{n}^{\prime \prime}(\varphi(z))\right| \\
\leq & (\Im z)^{\alpha}\left|\psi_{1}^{\prime}(z)\right| \max _{w \in K}\left|f_{n}(w)\right|+(\Im z)^{\alpha}\left|\psi_{1}(z) \varphi^{\prime}(z)+\psi_{2}^{\prime}(z)\right| \max _{w \in K}\left|f_{n}^{\prime}(w)\right| \\
& +(\Im z)^{\alpha}\left|\psi_{2}(z) \varphi^{\prime}(z)\right| \max _{w \in K}\left|f_{n}^{\prime \prime}(w)\right| \\
\leq & M_{1}(\Im \varphi(z))^{1 / p} \max _{w \in K}\left|f_{n}(w)\right|+M_{2}(\Im \varphi(z))^{1+1 / p} \max _{w \in K}\left|f_{n}^{\prime}(w)\right| \\
& +M_{3}(\Im \varphi(z))^{2+1 / p} \max _{w \in K}\left|f_{n}^{\prime \prime}(w)\right| \\
\leq & M_{1} \max _{w \in K}(\Im w)^{1 / p} \max _{w \in K}\left|f_{n}(w)\right|+M_{2} \max _{w \in K}(\Im w)^{1+1 / p} \max _{w \in K}\left|f_{n}^{\prime}(w)\right| \\
& +M_{3}(\Im w)^{2+1 / p} \max _{w \in K}\left|f_{n}^{\prime \prime}(w)\right| \\
< & 3 \varepsilon .
\end{aligned}
$$

Further, using (39), we have

$$
\left|T_{\psi_{1}, \psi_{2}, \varphi} f_{n}(i)\right|=\left|\psi_{1}(i) f_{n}(\varphi(i))+\psi_{2}(i) f_{n}^{\prime}(\varphi(i))\right| \leq\left(\left|\psi_{1}(i)\right|+\left|\psi_{2}(i)\right|\right) \varepsilon
$$

for $n \geq n_{0}$. 
From (40) and (41) it follows that

$$
\left\|T_{\psi_{1}, \psi_{2}, \varphi} f_{n}\right\|_{\mathcal{B}^{\alpha}\left(\Pi^{+}\right)}<\left(3+\left|\psi_{1}(i)\right|+\left|\psi_{2}(i)\right|\right) \varepsilon
$$

for $n \geq n_{0}$.

Since $\varepsilon>0$ is arbitrary, by Lemma 3 it follows that $T_{\psi_{1}, \psi_{2}, \varphi}: H^{p}\left(\Pi^{+}\right) \rightarrow \mathcal{B}^{\alpha}\left(\Pi^{+}\right)$is compact.

Conversely, suppose that $T_{\psi_{1}, \psi_{2}, \varphi}: H^{p}\left(\Pi^{+}\right) \rightarrow \mathcal{B}^{\alpha}\left(\Pi^{+}\right)$is compact. Then, clearly, it is bounded. Let $\left(z_{n}\right)_{n \in \mathbb{N}}$ be a sequence in $\Pi^{+}$such that $\varphi\left(z_{n}\right) \rightarrow \widehat{\partial \Pi^{+}}$as $n \rightarrow \infty$. If such a sequence does not exist, then conditions (i)-(iii) vacuously hold.

Let $w_{n}=\varphi\left(z_{n}\right), n \in \mathbb{N}$, and let the family of functions $\left(f_{n}\right)_{n \in \mathbb{N}}$ be defined by

$$
f_{n}(z)=\frac{\left(\Im w_{n}\right)^{2-1 / p}}{\pi^{1 / p}\left(z-\bar{w}_{n}\right)^{2}}-4 i \frac{\left(\Im w_{n}\right)^{3-1 / p}}{\pi^{1 / p}\left(z-\bar{w}_{n}\right)^{3}}-4 \frac{\left(\Im w_{n}\right)^{4-1 / p}}{\pi^{1 / p}\left(z-\bar{w}_{n}\right)^{4}}
$$

Then by using (22) and some simple estimates it is easy to see that $\left(f_{n}\right)_{n \in \mathbb{N}}$ weakly converges to zero as $n \rightarrow \infty$ (including the case when $w_{n} \rightarrow \infty$ as $n \rightarrow \infty$ ). Hence

$$
\lim _{n \rightarrow \infty}\left\|T_{\psi_{1}, \psi_{2}, \varphi} f_{n}\right\|_{\mathcal{B}^{\alpha}\left(\Pi^{+}\right)}=0
$$

The boundedness of $T_{\psi_{1}, \psi_{2}, \varphi}: H^{p}\left(\Pi^{+}\right) \rightarrow \mathcal{B}^{\alpha}\left(\Pi^{+}\right)$, together with (23), implies

$$
\begin{aligned}
\left\|T_{\psi_{1}, \psi_{2}, \varphi} f_{n}\right\|_{\mathcal{B}^{\alpha}\left(\Pi^{+}\right) \geq} & \left(\Im z_{n}\right)^{\alpha} \mid \psi_{1}^{\prime}\left(z_{n}\right) f_{n}\left(\varphi\left(z_{n}\right)\right)+\left(\psi_{1}\left(z_{n}\right) \varphi^{\prime}\left(z_{n}\right)+\psi_{2}^{\prime}\left(z_{n}\right)\right) f_{n}^{\prime}\left(\varphi\left(z_{n}\right)\right) \\
& +\psi_{2}\left(z_{n}\right) \varphi^{\prime}\left(z_{n}\right) f_{n}^{\prime \prime}\left(\varphi\left(z_{n}\right)\right) \mid \\
= & \frac{\left(\Im z_{n}\right)^{\alpha}}{8 \pi^{1 / p}\left(\Im \varphi\left(z_{n}\right)\right)^{2+1 / p}}\left|\psi_{2}\left(z_{n}\right) \varphi^{\prime}\left(z_{n}\right)\right| .
\end{aligned}
$$

From (42) and (43) we obtain

$$
\lim _{\varphi\left(z_{n}\right) \rightarrow \widehat{\partial \Pi^{+}}} \frac{\left(\Im z_{n}\right)^{\alpha}}{\left(\Im \varphi\left(z_{n}\right)\right)^{2+1 / p}}\left|\psi_{2}\left(z_{n}\right) \varphi^{\prime}\left(z_{n}\right)\right|=0
$$

from which it follows that condition (i) holds.

By considering families of functions

$$
g_{n}(z)=12 \frac{\left(\Im w_{n}\right)^{2-1 / p}}{\pi^{1 / p}\left(z-\bar{w}_{n}\right)^{2}}-32 i \frac{\left(\Im w_{n}\right)^{3-1 / p}}{\pi^{1 / p}\left(z-\bar{w}_{n}\right)^{3}}-24 \frac{\left(\Im w_{n}\right)^{4-1 / p}}{\pi^{1 / p}\left(z-\bar{w}_{n}\right)^{4}}
$$

and

$$
h_{n}(z)=8 \frac{\left(\Im w_{n}\right)^{2-1 / p}}{\pi^{1 / p}\left(z-\bar{w}_{n}\right)^{2}}-28 i \frac{\left(\Im w_{n}\right)^{3-1 / p}}{\pi^{1 / p}\left(z-\bar{w}_{n}\right)^{3}}-24 \frac{\left(\Im w_{n}\right)^{4-1 / p}}{\pi^{1 / p}\left(z-\bar{w}_{n}\right)^{4}}
$$

and proceeding as before, we can similarly show that

$$
\lim _{\varphi\left(z_{n}\right) \rightarrow \partial \Pi^{+}} \frac{\left(\Im z_{n}\right)^{\alpha}}{\left(\Im \varphi\left(z_{n}\right)\right)^{1+1 / p}}\left|\psi_{1}\left(z_{n}\right) \varphi^{\prime}\left(z_{n}\right)+\psi_{2}^{\prime}\left(z_{n}\right)\right|=0
$$


and

$$
\lim _{\varphi\left(z_{n}\right) \rightarrow \partial \widehat{\Pi}^{+}} \frac{\left(\Im z_{n}\right)^{\alpha}}{\left(\Im \varphi\left(z_{n}\right)\right)^{1 / p}}\left|\psi_{1}^{\prime}\left(z_{n}\right)\right|=0,
$$

from which it follows that conditions (ii) and (iii) hold, respectively.

Corollary 17 Let $1 \leq p<\infty, \alpha>0$ and $\varphi \in S\left(\Pi^{+}\right)$. Then $C_{\varphi}: H^{p}\left(\Pi^{+}\right) \rightarrow \mathcal{B}^{\alpha}\left(\Pi^{+}\right)$is compact if and only if it is bounded and

$$
\lim _{\varphi(z) \rightarrow \partial \Pi^{+}} \frac{(\Im z)^{\alpha}}{(\Im \varphi(z))^{1+1 / p}}\left|\varphi^{\prime}(z)\right|=0
$$

Corollary 18 Let $1 \leq p<\infty, \alpha>0, \psi \in H\left(\Pi^{+}\right)$and $\varphi \in S\left(\Pi^{+}\right)$. Then $M_{\psi} C_{\varphi}: H^{p}\left(\Pi^{+}\right) \rightarrow$ $\mathcal{B}^{\alpha}\left(\Pi^{+}\right)$is compact if and only if it is bounded and

$$
\begin{aligned}
& \text { (i) } \lim _{\varphi(z) \rightarrow \partial \widetilde{\Pi}^{+}} \frac{(\Im z)^{\alpha}}{(\Im \varphi(z))^{1 / p}}\left|\psi^{\prime}(z)\right|=0, \\
& \text { (ii) } \lim _{\varphi(z) \rightarrow \partial{\widetilde{\Pi^{+}}}^{+}} \frac{(\Im z)^{\alpha}}{(\Im \varphi(z))^{1+1 / p}}\left|\psi(z) \varphi^{\prime}(z)\right|=0 .
\end{aligned}
$$

Corollary 19 Let $1 \leq p<\infty, \alpha>0, \psi \in H\left(\Pi^{+}\right)$and $\varphi \in S\left(\Pi^{+}\right)$. Then $C_{\varphi} M_{\psi}: H^{p}\left(\Pi^{+}\right) \rightarrow$ $\mathcal{B}^{\alpha}\left(\Pi^{+}\right)$is compact if and only if it is bounded and

$$
\begin{aligned}
& \text { (i) } \lim _{\varphi(z) \rightarrow \partial \widehat{\Pi}^{+}} \frac{(\Im z)^{\alpha}}{(\Im \varphi(z))^{1 / p}}\left|\psi^{\prime}(\varphi(z)) \varphi^{\prime}(z)\right|=0, \\
& \text { (ii) } \quad \lim _{\varphi(z) \rightarrow \partial \widetilde{\Pi}^{+}} \frac{(\Im z)^{\alpha}}{(\Im \varphi(z))^{1+1 / p}}\left|\psi(\varphi(z)) \varphi^{\prime}(z)\right|=0 .
\end{aligned}
$$

Corollary 20 Let $1 \leq p<\infty, \alpha>0$ and $\varphi \in S\left(\Pi^{+}\right)$. Then $C_{\varphi} D: H^{p}\left(\Pi^{+}\right) \rightarrow \mathcal{B}^{\alpha}\left(\Pi^{+}\right)$is compact if and only if it is bounded and

$$
\lim _{\varphi(z) \rightarrow \partial \widetilde{\Pi}^{+}} \frac{(\Im z)^{\alpha}}{(\Im \varphi(z))^{2+1 / p}}\left|\varphi^{\prime}(z)\right|=0
$$

Corollary 21 Let $1 \leq p<\infty, \alpha>0$ and $\varphi \in S\left(\Pi^{+}\right)$. Then $D C_{\varphi}: H^{p}\left(\Pi^{+}\right) \rightarrow \mathcal{B}^{\alpha}\left(\Pi^{+}\right)$is compact if and only if it is bounded and

$$
\begin{aligned}
& \text { (i) } \lim _{\varphi(z) \rightarrow \partial{\Pi^{+}}^{+}} \frac{(\Im z)^{\alpha}}{(\Im \varphi(z))^{1+1 / p}}\left|\varphi^{\prime \prime}(z)\right|=0, \\
& \text { (ii) } \quad \lim _{\varphi(z) \rightarrow \partial \widetilde{\Pi}^{+}} \frac{(\Im z)^{\alpha}}{(\Im \varphi(z))^{2+1 / p}}\left|\varphi^{\prime}(z)\right|^{2}=0 .
\end{aligned}
$$

Corollary 22 Let $1 \leq p<\infty, \alpha>0, \psi \in H\left(\Pi^{+}\right)$and $\varphi \in S\left(\Pi^{+}\right)$. Then $M_{\psi} C_{\varphi} D: H^{p}\left(\Pi^{+}\right) \rightarrow$ $\mathcal{B}^{\alpha}\left(\Pi^{+}\right)$is compact if and only if it is bounded and
(i) $\lim _{\varphi(z) \rightarrow \widehat{\partial \Pi}^{+}} \frac{(\Im z)^{\alpha}}{(\Im \varphi(z))^{1+1 / p}}\left|\psi^{\prime}(z)\right|=0$,
(ii) $\lim _{\varphi(z) \rightarrow \partial \widetilde{\Pi}^{+}} \frac{(\Im z)^{\alpha}}{(\Im \varphi(z))^{2+1 / p}}\left|\psi(z) \varphi^{\prime}(z)\right|=0$. 
Corollary 23 Let $1 \leq p<\infty, \alpha>0, \psi \in H\left(\Pi^{+}\right)$and $\varphi \in S\left(\Pi^{+}\right)$. Then $C_{\varphi} M_{\psi} D: H^{p}\left(\Pi^{+}\right) \rightarrow$ $\mathcal{B}^{\alpha}\left(\Pi^{+}\right)$is compact if and only if it is bounded and

(i) $\lim _{\varphi(z) \rightarrow \partial \Pi^{+}} \frac{(\Im z)^{\alpha}}{(\Im \varphi(z))^{1+1 / p}}\left|\psi^{\prime}(\varphi(z)) \varphi^{\prime}(z)\right|=0$

(ii) $\lim _{\varphi(z) \rightarrow \partial \widetilde{\Pi}^{+}} \frac{(\Im z)^{\alpha}}{(\Im \varphi(z))^{2+1 / p}}\left|\psi(\varphi(z)) \varphi^{\prime}(z)\right|=0$.

Corollary 24 Let $1 \leq p<\infty, \alpha>0, \psi \in H\left(\Pi^{+}\right)$and $\varphi \in S\left(\Pi^{+}\right)$. Then $M_{\psi} D C_{\varphi}: H^{p}\left(\Pi^{+}\right) \rightarrow$ $\mathcal{B}^{\alpha}\left(\Pi^{+}\right)$is compact if and only if it is bounded and
(i) $\lim _{\varphi(z) \rightarrow \widetilde{\partial \Pi^{+}}} \frac{(\Im z)^{\alpha}}{(\Im \varphi(z))^{1+1 / p}}\left|\left(\psi \varphi^{\prime}\right)^{\prime}(z)\right|=0$,
(ii) $\lim _{\varphi(z) \rightarrow \partial \widetilde{\Pi}^{+}} \frac{(\Im z)^{\alpha}}{(\Im \varphi(z))^{2+1 / p}}\left|\psi(z)\left(\varphi^{\prime}(z)\right)^{2}\right|=0$.

Corollary 25 Let $1 \leq p<\infty, \alpha>0, \psi \in H\left(\Pi^{+}\right)$and $\varphi \in S\left(\Pi^{+}\right)$. Then $D M_{\psi} C_{\varphi}: H^{p}\left(\Pi^{+}\right) \rightarrow$ $\mathcal{B}^{\alpha}\left(\Pi^{+}\right)$is compact if and only if it is bounded and
(i) $\lim _{\varphi(z) \rightarrow \partial{\Pi^{+}}^{+}} \frac{(\Im z)^{\alpha}}{(\Im \varphi(z))^{1 / p}}\left|\psi^{\prime \prime}(z)\right|=0$,
(ii) $\lim _{\varphi(z) \rightarrow \partial \widehat{\Pi}^{+}} \frac{(\Im z)^{\alpha}}{(\Im \varphi(z))^{1+1 / p}}\left|2 \psi^{\prime}(z) \varphi^{\prime}(z)+\psi(z) \varphi^{\prime \prime}(z)\right|=0$,
(iii) $\lim _{\varphi(z) \rightarrow \partial \Pi^{+}} \frac{(\Im z)^{\alpha}}{(\Im \varphi(z))^{2+1 / p}}\left|\psi(z)\left(\varphi^{\prime}(z)\right)^{2}\right|=0$

Corollary 26 Let $1 \leq p<\infty, \alpha>0, \psi \in H\left(\Pi^{+}\right)$and $\varphi \in S\left(\Pi^{+}\right)$. Then $C_{\varphi} D M_{\psi}: H^{p}\left(\Pi^{+}\right) \rightarrow$ $\mathcal{B}^{\alpha}\left(\Pi^{+}\right)$is compact if and only if it is bounded and
(i) $\lim _{\varphi(z) \rightarrow \partial \widetilde{\Pi}^{+}} \frac{(\Im z)^{\alpha}}{(\Im \varphi(z))^{1 / p}}\left|\psi^{\prime \prime}(\varphi(z)) \varphi^{\prime}(z)\right|=0$,
(ii) $\lim _{\varphi(z) \rightarrow \widetilde{\Pi^{+}}} \frac{(\Im z)^{\alpha}}{(\Im \varphi(z))^{1+1 / p}}\left|\psi^{\prime}(\varphi(z)) \varphi^{\prime}(z)\right|=0$,
(iii) $\lim _{\varphi(z) \rightarrow \partial{\Pi^{+}}^{+}} \frac{(\Im z)^{\alpha}}{(\Im \varphi(z))^{2+1 / p}}\left|\psi(\varphi(z)) \varphi^{\prime}(z)\right|=0$.

Corollary 27 Let $1 \leq p<\infty, \alpha>0, \psi \in H\left(\Pi^{+}\right)$and $\varphi \in S\left(\Pi^{+}\right)$. Then $D C_{\varphi} M_{\psi}: H^{p}\left(\Pi^{+}\right) \rightarrow$ $\mathcal{B}^{\alpha}\left(\Pi^{+}\right)$is compact if and only if it is bounded and
(i) $\lim _{\varphi(z) \rightarrow \overparen{\partial \Pi^{+}}} \frac{(\Im z)^{\alpha}}{(\Im \varphi(z))^{1 / p}}\left|\psi^{\prime}(\varphi(z)) \varphi^{\prime \prime}(z)+\psi^{\prime \prime}(\varphi(z))\left(\varphi^{\prime}(z)\right)^{2}\right|=0$,
(ii) $\lim _{\varphi(z) \rightarrow \partial{\widetilde{\Pi^{+}}}^{+}} \frac{(\Im z)^{\alpha}}{(\Im \varphi(z))^{1+1 / p}}\left|2 \psi^{\prime}(\varphi(z))\left(\varphi^{\prime}(z)\right)^{2}+\psi(\varphi(z)) \varphi^{\prime \prime}(z)\right|=0$,
(iii) $\lim _{\varphi(z) \rightarrow \widetilde{\Pi^{+}}} \frac{(\Im z)^{\alpha}}{(\Im \varphi(z))^{2+1 / p}}\left|\psi(\varphi(z))\left(\varphi^{\prime}(z)\right)^{2}\right|=0$. 


\section{Acknowledgements}

The study in the paper is a part of the investigation at the projects III 41025 and III 44006 by the Serbian Ministry of Education and Science.

Funding

Not applicable.

Availability of data and materials

Not applicable.

\section{Competing interests}

The authors declare that they have no competing interests.

Authors' contributions

The authors have contributed equally to the writing of this paper. They read and approved the manuscript.

\section{Author details}

'Mathematical Institute of the Serbian Academy of Sciences, Beograd, Serbia. ${ }^{2}$ Department of Medical Research, China Medical University Hospital, China Medical University, Taichung, Taiwan, Republic of China. ${ }^{3}$ Department of Mathematics, Central University of Jammu, Jammu, India.

\section{Publisher's Note}

Springer Nature remains neutral with regard to jurisdictional claims in published maps and institutional affiliations.

Received: 10 August 2018 Accepted: 30 September 2018 Published online: 10 October 2018

\section{References}

1. Avetisyan, K.L.: Hardy-Bloch type spaces and lacunary series on the polydisk. Glasg. Math. J. 49(2), 345-356 (2007)

2. Avetisyan, K.L.: Weighted integrals and Bloch spaces of $n$-harmonic functions on the polydisc. Potential Anal. 29(1), 49-63 (2008)

3. Benke, G., Chang, D.C.: A note on weighted Bergman spaces and the Cesáro operator. Nagoya Math. J. 159, 25-43 (2000)

4. Jiang, Z.J.: Generalized product-type operators from weighted Bergman-Orlicz spaces to Bloch-Orlicz spaces. Appl. Math. Comput. 268, 966-977 (2015)

5. Jiang, Z.J.: On Stević-Sharma operator from the Zygmund space to the Bloch-Orlicz space. Adv. Differ. Equ. 2015, Article ID 228 (2015)

6. Li, H., Guo, Z: On a product-type operator from Zygmund-type spaces to Bloch-Orlicz spaces. J. Inequal. Appl. 2015, Article ID 132 (2015)

7. Li, S., Stević, S.: Composition followed by differentiation from mixed-norm spaces to $\alpha$-Bloch spaces. Sb. Math. 199(12), 1847-1857 (2008)

8. Li, S., Stević, S.: Products of composition and differentiation operators from Zygmund spaces to Bloch spaces and Bers spaces. Appl. Math. Comput. 217, 3144-3154 (2010)

9. Li, S., Stević, S.: Weighted differentiation composition operators from the logarithmic Bloch space to the weighted-type space. An. Ştiinţ. Univ. 'Ovidius' Constanţa, Ser. Mat. 24(3), 223-240 (2016)

10. Sehba, B., Stević, S.: On some product-type operators from Hardy-Orlicz and Bergman-Orlicz spaces to weighted-type spaces. Appl. Math. Comput. 233C, 565-581 (2014)

11. Stević, S.: Characterizations of composition followed by differentiation between Bloch-type spaces. Appl. Math. Comput. 218, 4312-4316 (2011)

12. Zhu, X.: Generalized weighted composition operators from Bloch-type spaces to weighted Bergman spaces. Indian J. Math. 49(2), 139-149 (2007)

13. Zhu, X:: Essential norm and compactness of the product of differentiation and composition operators on Bloch type spaces. Math. Inequal. Appl. 19(1), 325-334 (2016)

14. Stević, S.: Composition operators from the Hardy space to the Zygmund-type space on the upper half-plane. Abstr. Appl. Anal. 2009, Article ID 161528 (2009)

15. Stević, S.: Composition operators from the Hardy space to the $n$th weighted-type space on the unit disk and the half-plane. Appl. Math. Comput. 215, 3950-3955 (2010)

16. Stević, S.: Essential norm of some extensions of the generalized composition operators between kth weighted-type spaces. J. Inequal. Appl. 2017, Article ID 220 (2017)

17. Matache, V:: Composition operators on $H^{p}$ of the upper half-plane. Proc. An. Univ. Timisoara Ser. Stiint. Mat. 27, 63-66 (1989)

18. Matache, V.: Compact composition operators on $H^{p}$ of a half-plane. Proc. Am. Math. Soc. 127, 1483-1491 (1999)

19. Sharma, S.D.: Compact and Hilbert-Schmidt composition operators on Hardy spaces of the upper half-plane. Acta Sci. Math. 46(1-4), 197-202 (1983)

20. Singh, R.K., Sharma, S.D.: Non-compact composition operators. Bull. Aust. Math. Soc. 21, 125-130 (1980)

21. Sharma, S.D., Sharma, A.K., Ahmed, S.: Composition operators between Hardy and Bloch-type spaces of the upper half-plane. Bull. Korean Math. Soc. 44, 475-482 (2007)

22. Sharma, A.K., Ueki, S.: Compact composition operators on the Bloch space and the growth space of the upper half-plane. Mediterr. J. Math. 14(2), Article ID 76 (2017)

23. Stević, S., Sharma, A.K.: Weighted composition operators between growth spaces of the upper half-plane. Util. Math. $84,265-272(2011)$ 
24. Stević, S., Sharma, A.K.: Weighted composition operators between Hardy and growth spaces on the upper half-plane. Appl. Math. Comput. 217, 4928-4934 (2011)

25. Stević, S., Sharma, A.K., Sharma, S.D.: Weighted composition operators from weighted Bergman spaces to weighted-type spaces on the upper half-plane. Abstr. Appl. Anal. 2011, Article ID 989625 (2011)

26. Li, S., Stević, S.: Composition followed by differentiation between $H^{\infty}$ and $\alpha$-Bloch spaces. Houst. J. Math. 35 , 327-340 (2009)

27. Ohno, S.: Products of composition and differentiation between Hardy spaces. Bull. Aust. Math. Soc. 73(2), 235-243 (2006)

28. Yang, W.: Products of composition and differentiation operators from $\mathcal{Q}_{k}(p, q)$ spaces to Bloch-type spaces. Abstr. Appl. Anal. 2009, Article ID 741920 (2009)

29. Zhou, J., Zhu, X.: Product of differentiation and composition operators on the logarithmic Bloch space. J. Inequal. Appl. 2014, Article ID 453 (2014)

30. Stević, S.: Weighted differentiation composition operators from mixed-norm spaces to weighted-type spaces. Appl. Math. Comput. 211, 222-233 (2009)

31. Yang, W.: Generalized weighted composition operators from the $F(p, q, s)$ space to the Bloch-type space. Appl. Math. Comput. 218, 4967-4972 (2012)

32. Yu, Y., Liu, Y.: The essential norms of a generalized composition operators between Bloch type spaces and $Q_{k}$ type spaces. Complex Anal. Oper. Theory 6(6), 1231-1240 (2012)

33. Zhu, X:: Essential norm of generalized weighted composition operators on Bloch-type spaces. Appl. Math. Comput. $274,133-142(2016)$

34. Li, S., Stević, S.: Products of composition and integral type operators from $H^{\infty}$ to the Bloch space. Complex Var. Elliptic Equ. 53(5), 463-474 (2008)

35. Li, H., Li, S.: Norm of an integral operator on some analytic function spaces on the unit disk. J. Inequal. Appl. 2013, Article ID 342 (2013)

36. Ueki, S.I.: On the Li-Stević integral type operators from weighted Bergman spaces into $\beta$-Zygmund spaces. Integral Equ. Oper. Theory 74(1), 137-150 (2012)

37. Yang, W.: Volterra composition operators from $F(p, q, s)$ spaces to Bloch-type spaces. Bull. Malays. Math. Sci. Soc. 34(2), 267-277 (2011)

38. Yu, Y, Liu, Y.: On Li-Stević integral-type operators between different weighted Bloch-type spaces. J. Inequal. Appl. 2008, Article ID 780845 (2008)

39. Zhu, X.: Volterra composition operators from weighted-type spaces to Bloch-type spaces and mixed norm spaces. Math. Inequal. Appl. 14(1), 223-233 (2011)

40. Zhu, X.: Generalized Riemann-Stieltjes operators between different weighted Bergman spaces. J. Comput. Anal. Appl. 14(3), 426-432 (2012)

41. Jiang, Z.J.: On a product operator from weighted Bergman-Nevanlinna spaces to weighted Zygmund spaces J. Inequal. Appl. 2014, Article ID 404 (2014)

42. Liu, Y., Yu, Y.: On a Stević-Sharma operator from Hardy spaces to the logarithmic Bloch spaces. J. Inequal. Appl. 2014, Article ID 22 (2014)

43. Sharma, A.K.: Products of multiplication, composition and differentiation between weighted Bergman-Nevanlinna and Bloch-type spaces. Turk. J. Math. 35, 275-291 (2011)

44. Stević, S.: On operator $P_{\varphi}^{g}$ from the logarithmic Bloch-type space to the mixed-norm space on unit ball. Appl. Math. Comput. 215, 4248-4255 (2010)

45. Stević, S., Sharma, A.K., Bhat, A.: Products of multiplication composition and differentiation operators on weighted Bergman spaces. Appl. Math. Comput. 217, 8115-8125 (2011)

46. Stević, S., Sharma, A.K., Bhat, A.: Essential norm of products of multiplication composition and differentiation operators on weighted Bergman spaces. Appl. Math. Comput. 218, 2386-2397 (2011)

47. Schwartz, H.J.: Composition operators on $H^{p}$. Thesis, University of Toledo (1969)

\section{Submit your manuscript to a SpringerOpen ${ }^{\circ}$ journal and benefit from:}

- Convenient online submission

- Rigorous peer review

- Open access: articles freely available online

- High visibility within the field

- Retaining the copyright to your article

Submit your next manuscript at $\gg$ springeropen.com 